\title{
Limited Efficacy of Endoscopic Third Ventriculostomy for Hydrocephalus Following Aneurysmal Subarachnoid Hemorrhage
}

\author{
Toru FUKUHARA, Tomohisa SHIMIZU, and Yoichiro NAMBA \\ Division of Neurosurgery, Neuro-research Institute for Stroke Care, \\ National Hospital Organization Okayama Medical Center, Okayama
}

\begin{abstract}
Endoscopic third ventriculostomy (ETV) has not been recognized as a surgical option for hydrocephalus following aneurysmal subarachnoid hemorrhage (SAH), since hydrocephalus following aneurysmal SAH is considered to result from the communicating component. However, obstructions in the ventricular system may exist, so ETV could help resolve the condition. The present study evaluated the efficacy of ETV for hydrocephalus appearing within one month after aneurysmal SAH. This prospective study evaluated a total of 118 patients admitted to our hospital with aneurysmal SAH. Nine of 66 surgically treated patients suffered hydrocephalus within one month after aneurysmal SAH and 8 underwent ETV. Seven of these 8 patients showed no further ventricular enlargement or deterioration in consciousness, and required no external cerebrospinal fluid (CSF) drainage at least temporarily, and could commence early physical rehabilitation. Four patients also experienced cognitive improvements after ETV, but none made a full cognitive recovery. Ventriculoperitoneal (VP) shunt was implanted for one patient who did not respond to ETV, and the necessity of VP shunt was evaluated including the CSF removal test for the other patients, due to residual cognitive impairment even after initiating the rehabilitation. Five of the 8 patients eventually had VP shunts implanted, and 3 patients, including two patients who improved cognitively after ETV, had further cognitive improvements. ETV for hydrocephalus following aneurysmal SAH is likely to help manage intracranial pressure. ETV may improve cognitive impairment in some patients, but whether the maximum resolution is obtained only with ETV remains uncertain. VP shunt implantation should be the main treatment for hydrocephalus after aneurysmal SAH, but ETV can be employed as a temporary intervention in certain conditions, such as during the waiting period for the clearance of aneurysmal SAH.
\end{abstract}

Key words: endoscopic third ventriculostomy, hydrocephalus, intracranial pressure, subarachnoid hemorrhage, ventriculoperitoneal shunt

\section{Introduction}

Hydrocephalus is a complication of aneurysmal subarachnoid hemorrhage $(\mathrm{SAH})$ in $10-30 \%{ }^{6)}$ or $6-67 \%{ }^{4)}$ of patients, and approximately $20 \%$ of all such patients ultimately develop shunt-dependent hydrocephalus. ${ }^{16)}$ Treatment generally depends on implantation of a ventriculoperitoneal (VP) shunt, but opening of the lamina terminalis has been used successfully in combination with aneurysm clipping to prevent the development of hydrocephalus following aneurysmal SAH. ${ }^{8,15)}$ Impaired cerebrospinal fluid (CSF) absorption at the arachnoid granules due

Received August 11, 2008; Accepted March 18, 2009 to fibrosis is generally accepted as a mechanism of hydrocephalus following aneurysmal $\mathrm{SAH},{ }^{16)}$ but obstruction of the CSF circulation within the ventricular system is also important, ${ }^{4)}$ since intraventricular hemorrhage (IVH) at initial computed tomography (CT) is a significant predictor of hydrocephalus following aneurysmal SAH. ${ }^{4,12,16)}$ The preventive effect of lamina terminalis fenestration at clipping surgery ${ }^{8,15)}$ also indicates that CSF flow obstruction within the ventricular system must be a significant factor. In some cases, the obstructed portion of CSF flow after aneurysmal SAH may be located in the ventricular system, and opening the lamina terminalis can decrease the rate of hydrocephalus occurrence. Therefore, endoscopic third ventriculostomy (ETV) may be an effective treatment of 
hydrocephalus following aneurysmal SAH.

The present study summarizes our experience with ETV for the treatment of hydrocephalus appearing within one month after aneurysmal SAH, and evaluates the effectiveness and limitations.

\section{Clinical Materials and Methods}

The treatment protocol for aneurysmal SAH in our institute requires that patients with incidental nontraumatic SAH are scheduled for emergent conventional angiography within 6 hours of admission, except for patients in Hunt and Kosnik grade $\mathrm{V}$ or patients older than 80 years old, who are treated conservatively. If angiography confirms the presence of a ruptured aneurysm, surgery is performed within 24 hours, after excluding any cardiac problems affecting the induction of general anesthesia. If no origin for the SAH is identified, threedimensional CT angiography is performed the next day, and angiography is repeated at least twice. If a ruptured aneurysm is detected, surgery is scheduled within 72 hours of admission if possible or delayed until 2 weeks after admission. We generally use endovascular intervention for posterior circulation aneurysms, and even for some anterior circulation aneurysms, depending on the general condition such as the use of anticoagulant agents. External cisternal and/or external ventricular drainage (EVD) is placed in all open craniotomies, and promptly after the endovascular coiling procedure. No drainage is placed in patients with minimal SAH or undergoing delayed surgery due to initially unknown origin or cardiac problems on admission. After surgery, continuous administration of intravenous diltiazem hydrochloride and ozagrel sodium, and three times daily injection of fasudil hydrochloride are given for 2 weeks to prevent vasospasm. The EVD is removed after confirming the correct functioning of the cisternal drainage, but the EVD is retained if functioning of the cisternal drainage is inadequate.

Our treatment protocol for hydrocephalus following SAH consists of continuous CSF drainage for 3 days, then gradual increase of the height of the chamber, and attempted removal of drainage within 7 days. However, if ventricular enlargement or deterioration of consciousness occurs while clamping the drainage, the drainage will be kept in place for another week at the height of $20 \mathrm{cmH}_{2} \mathrm{O}$ from the external auditory meatus. In our institute, VP shunt implantation is postponed for at least one month after aneurysm rupture to achieve better shunt patency. If external CSF drainage is still necessary within a month of an aneurysmal SAH event, then reinstal- lation of the EVD will be considered. This study regards this condition as hydrocephalus occurring within one month after aneurysmal SAH, and recognizes two modes: Enlarged ventricles occurring within one month after drain removal with consciousness disturbance; and continued flow of more than $200 \mathrm{ml} /$ day of CSF at 2 weeks after aneurysm rupture preventing drain removal. Both conditions are considered to be high risk without external CSF drainage, and thus indicate prompt reinstallation of the EVD.

In the explanation of treatment to the patient's family, the option of ETV is discussed. The advantage is possible resolution of hydrocephalus without the need for external drainage of CSF, although the success rate is unknown. However, ETV carries the surgical risk of third ventriculostomy but risks related to general anesthesia, since EVD can be placed under local anesthesia. We emphasize that VP shunt implantation will probably be necessary if hydrocephalus persists even after ETV. If the patient's family wishes to proceed, ETV is performed at the reinstallation of EVD. An intracranial pressure (ICP) monitoring system is also placed if available, and involves preoperative implantation of the ICP sensor at the bedside for measuring ICP overnight, and continuous monitoring for 3 days postoperatively. The EVD will not be opened unless clinical signs indicate increased ICP. This clinical study was approved as institutionally funded research in April 2002.

A total of 118 patients with nontraumatic incidental SAH were admitted to our institute between April 2002 and March 2006. No surgical treatment was recommended for 46 patients because of grade $\mathrm{V}$ on admission (23 patients), older than 80 years (14 patients), or unknown origin even after repeated cerebral angiography (9 patients). Clipping surgery could not be completed due to cardiac problems in 6 patients ( 4 acute myocardial infarction and 2 ampulla cardiomyopathy), which lead to their deaths. Sixty-six patients were treated surgically (59 clipping and 7 coiling), but no CSF drainage was placed in 7 patients because of minimal aneurysmal SAH or aneurysmal SAH already washed out due to the delayed intervention. Four of the 59 patients who had CSF drainage at the time of aneurysm surgery died within 2 weeks of the surgery due to uncontrollable increases in ICP. Nine patients developed hydrocephalus within one month after aneurysmal $\mathrm{SAH}$ to the extent that replacement of the EVD seemed necessary. Hydrocephalus was due to enlarged ventricles in 5 patients, and drain removal failure in 4. The option of ETV at EVD replacement was explained to the families of these 9 patients. The 
family of a patient with unruptured basilar artery aneurysm refused this option due to the considerably higher risk of ETV. The remaining 8 patients received ETV at replacement of the EVD. Figure 1 shows the flow chart detailing how the study population was selected. Clinical features and outcomes, including the effect of ETV and successive VP shunt

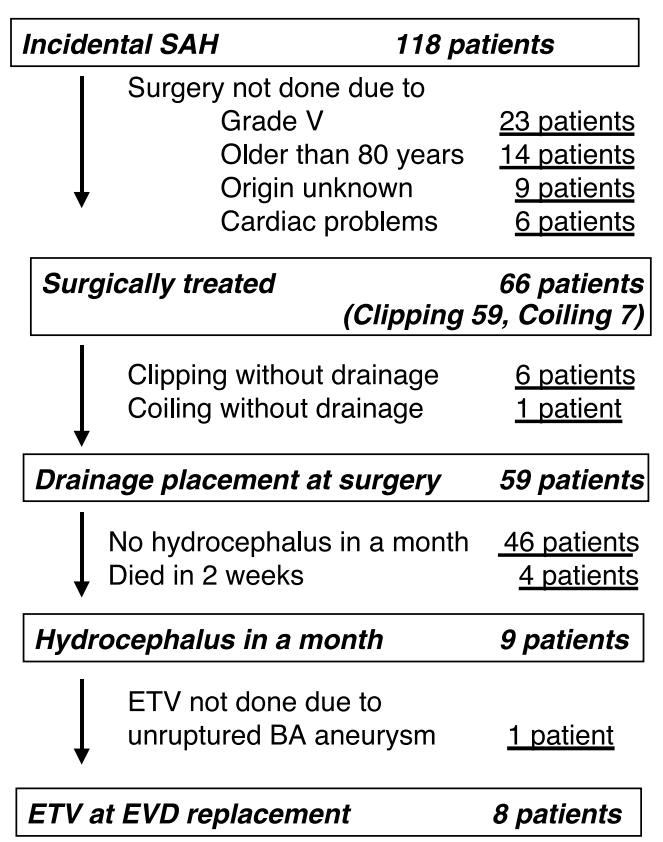

Fig. 1 Flow chart detailing selection of the eight patients. BA: basilar artery, ETV: endoscopic third ventriculostomy, EVD: external ventricular drainage, SAH: subarachnoid hemorrhage. implantation, if performed, were evaluated.

\section{Results}

The rate of hydrocephalus occurring within a month was calculated as $14.5 \%$, based on 9 of 62 surgically treated patients surviving at one month after aneurysmal SAH. However, the total incidence of hydrocephalus was $34.4 \%$ in patients treated with VP shunt implantation and/or ETV within 6 months, based on 21 of 61 surgically treated patients surviving at 6 months after aneurysmal SAH. The clinical features of the 8 patients who underwent ETV for hydrocephalus following aneurysmal SAH are described in Table 1. Patients were older at 51-79 years old, and the grade at presentation tended to be high, with 5 patients in grade IV. CT revealed thick clots in all patients except one (Case 2), who had a diffuse thin clot but also IVH in the fourth ventricle. Therefore, all patients had aneurysmal SAH corresponding to Fisher grade 3 or 4 . One patient with an aneurysm localized on the posterior circulation (Case 1) underwent intravascular embolization, and another patient with a left posterior communicating artery aneurysm (Case 8) also underwent embolization, since she had been receiving oral warfarin for prior venous thrombosis. Symptomatic spasms occurred after surgery in 3 patients.

Table 2 summarizes the results of ETV and patient outcomes. The external CSF drainage could be removed in 5 patients, but progressive enlargement of the ventricles was observed together with consciousness disturbance, so the EVD was replaced. The CSF drainage could not be removed 2 weeks after aneurysm rupture in the other 3 patients, so replace-

Table 1 Clinical features of eight patients undergoing endoscopic third ventriculostomy for hydrocephalus following aneurysmal subarachnoid hemorrhage

\begin{tabular}{|c|c|c|c|c|c|c|c|c|}
\hline $\begin{array}{l}\text { Case } \\
\text { No. }\end{array}$ & $\begin{array}{c}\text { Age } \\
\text { (yrs), } \\
\text { Sex }\end{array}$ & $\begin{array}{l}\text { Aneurysm } \\
\text { location }\end{array}$ & $\begin{array}{l}\text { H\&K } \\
\text { grade }\end{array}$ & $\begin{array}{l}\text { Cisternal } \\
\text { clot } \\
\text { thickness }\end{array}$ & $\begin{array}{l}\text { Extension } \\
\text { of IVH }\end{array}$ & $\begin{array}{l}\text { Location } \\
\text { of IPH }\end{array}$ & $\begin{array}{c}\text { Aneurysm } \\
\text { surgery }\end{array}$ & $\begin{array}{l}\text { Symptomatic } \\
\text { spasm }\end{array}$ \\
\hline 1 & $51, \mathrm{M}$ & lt PICA & IV & diffuse thick & lateral ventricle & none & coiling & none \\
\hline 2 & $75, \mathrm{~F}$ & ACoA & IV & diffuse thin & fourth ventricle & none & clipping & none \\
\hline 3 & $59, \mathrm{~F}$ & rt MCA & III & diffuse thick & none & none & clipping & aphasia \\
\hline 4 & $72, \mathrm{~F}$ & $\mathrm{ACoA}$ & IV & diffuse thick & none & none & clipping & $\begin{array}{l}\text { aphasia, } \\
\text { rt hemiplegia }\end{array}$ \\
\hline 5 & $79, \mathrm{~F}$ & lt MCA & IV & focal thick & lateral ventricle & lt putamen & clipping & none \\
\hline 6 & $70, \mathrm{~F}$ & lt PCoA & III & diffuse thick & third ventricle & none & clipping & none \\
\hline 7 & $63, \mathrm{M}$ & rt MCA & IV & focal thick & lateral ventricle & rt putamen & clipping & non \\
\hline 8 & $79, \mathrm{~F}$ & lt PCoA & II & diffuse thick & none & none & coiling & $\begin{array}{l}\text { aphasia, } \\
\text { rt hemiplegia }\end{array}$ \\
\hline
\end{tabular}

ACoA: anterior communicating artery, H\&K: Hunt \& Kosnik, IPH: intraparenchymal hemorrhage, IVH: intraventricular hemorrhage, MCA: middle cerebral artery, PCoA: posterior communicating artery, PICA: posterior inferior cerebellar artery. 
Table 2 Results of endoscopic third ventriculostomy (ETV) and outcomes of eight patients with hydrocephalus following aneurysmal subarachnoid hemorrhage

\begin{tabular}{|c|c|c|c|c|c|c|c|c|c|c|}
\hline \multirow{2}{*}{$\begin{array}{l}\text { Case } \\
\text { No. }\end{array}$} & \multirow{2}{*}{$\begin{array}{l}\text { Indication } \\
\text { of ETV }\end{array}$} & \multirow{2}{*}{$\begin{array}{l}\text { Admission } \\
\text { to ETV } \\
\text { (days) }\end{array}$} & \multirow{2}{*}{$\begin{array}{l}\text { Anesthesia } \\
\text { of ETV }\end{array}$} & \multicolumn{3}{|c|}{ Results of ETV in one week } & \multirow{2}{*}{$\begin{array}{l}\text { VP shunt } \\
\text { implanta- } \\
\text { tion }\end{array}$} & \multirow{2}{*}{$\begin{array}{l}\text { ETV to } \\
\text { VP shunt } \\
\text { (days) }\end{array}$} & \multirow{2}{*}{$\begin{array}{c}\text { Improvement } \\
\text { after } \\
\text { VP shunt }\end{array}$} & \multirow{2}{*}{$\begin{array}{l}\text { GOS at } \\
6 \mathrm{mos}\end{array}$} \\
\hline & & & & Ventricles & $\begin{array}{l}\text { Conscious- } \\
\text { ness }\end{array}$ & Cognition & & & & \\
\hline 1 & $\begin{array}{l}\text { enlarged } \\
\text { ventricles }\end{array}$ & 20 & general & stable & improved & improved & yes & 14 & yes & GR \\
\hline 2 & $\begin{array}{l}\text { enlarged } \\
\text { ventricles }\end{array}$ & 26 & general & reduced & improved & improved & yes & 28 & no & MD \\
\hline 3 & $\begin{array}{l}\text { enlarged } \\
\text { ventricles }\end{array}$ & 24 & general & stable & improved & improved & yes & 35 & yes & GR \\
\hline 4 & $\begin{array}{l}\text { enlarged } \\
\text { ventricles }\end{array}$ & 29 & general & stable & improved & no change & yes & 45 & no & SD \\
\hline 5 & $\begin{array}{l}\text { enlarged } \\
\text { ventricles }\end{array}$ & 22 & general & stable & no change & no change & no & NA & NA & VS \\
\hline 6 & $\begin{array}{l}\text { drain removal } \\
\text { failure }\end{array}$ & 20 & local & reduced & improved & improved & no & NA & NA & death \\
\hline 7 & $\begin{array}{l}\text { drain removal } \\
\text { failure }\end{array}$ & 17 & local & stable & no change & no change & no & NA & NA & VS \\
\hline 8 & $\begin{array}{l}\text { drain removal } \\
\text { failure }\end{array}$ & 15 & local & enlarged & no change & no change & yes & 69 & yes & SD \\
\hline
\end{tabular}

GOS: Glasgow Outcome Scale, GR: good recovery, MD: moderate disability, NA: not applicable, SD: severe disability, VP: ventriculoperitoneal, VS: vegetative state.
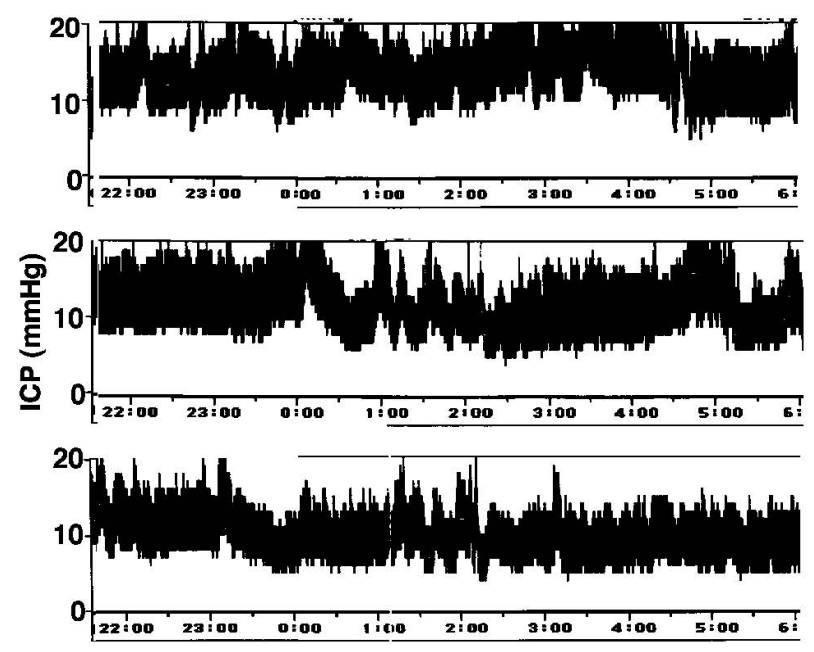

Fig. 2 Intracranial pressure (ICP) monitoring traces overnight in Case 2. Continuous ICP values were obtained on the day before endoscopic third ventriculostomy (ETV) (upper), the first night after ETV (middle), and the second night (lower), and showed reduction of ICP.

ment of EVD was performed. ETV was performed under general anesthesia in the earlier 5 patients, but local anesthesia was used without technical difficulties in the most recent 3 patients. Ventricular enlargement ceased after ETV (stable or decreased) in all patients except Case 8, so the EVD could be removed without draining CSF. ICP was monitored in
Cases 2, 3, and 7, revealing reduction compared with preoperative values (Fig. 2). Improvement in consciousness (e.g., eye opening or motor reactions to verbal stimulation) was seen in 5 patients, and cognitive recovery (e.g., appropriate reactions to verbal commands) in 4 patients.

Ventricular enlargement continued in Case 8 even after ETV, so continuous CSF drainage was necessary. Case 8 was the only patient who was unresponsive to ETV. VP shunt implantation was postponed, due to a prolonged serious skin rash caused by allergic reactions to antibiotics, so lumbar drainage was placed for 2 months. The patient's consciousness improved immediately after the VP shunt was implanted. However, her cognition was seriously impaired, so she was dependent in all her daily activities (severe disability on Glasgow Outcome Scale) despite intense rehabilitation. Case 6 responded to ETV quite well, so rehabilitation was initiated within a week, but she suffered lung embolization at the very beginning of standing rehabilitation, and eventually died.

VP shunt implantation was considered in the other 6 patients, since all had some level of cognitive impairment and enlarged ventricles even after ETV. Cases 5 and 7 with large intraparenchymal hemorrhages (IPHs) on admission did not exhibit improved consciousness, although no further ventricular enlargement occurred after ETV. Significant recovery after VP shunt implantation was quite unlikely with such large IPHs, so CSF removal tests were performed to assess the prognosis for VP shunt implan- 


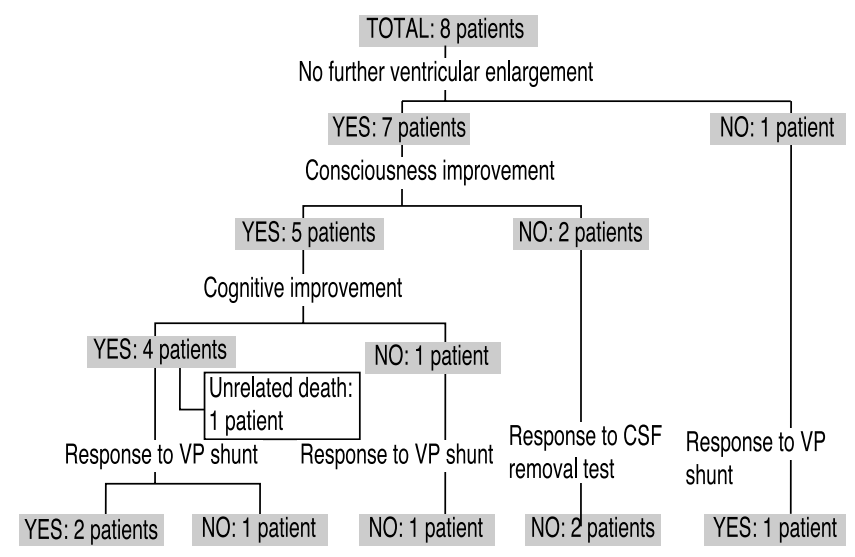

Fig. 3 Flow chart detailing the efficacy of endoscopic third ventriculostomy for hydrocephalus following aneurysmal subarachnoid hemorrhage. CSF: cerebrospinal fluid, VP: ventriculoperitoneal.

tation. Since no response was seen to the CSF removal test, VP shunt implantation was not performed. Cases 1-4 underwent VP shunt implantation. Cases 1 and 3 responded quickly and cognition had recovered completely at the 6 month follow-up evaluation. Cases 2 and 4 were considered nonresponders to VP shunt implantation because of the absence of signs of improvement. The responses to ETV and VP shunt implantation are summarized in Fig. 3.

\section{Discussion}

In our institute, VP shunt implantation is generally postponed until at least one month has passed to improve VP shunt patency. Prior to this study, we usually only replaced the EVD if hydrocephalus appeared within a month after aneurysmal SAH. The present clinical study compared the advantages of ETV against the added risk of the ETV procedure. We found no operative complications with ETV, so we believe the benefits outweigh the possible harm.

This study only examined hydrocephalus appearing within a month after aneurysmal $\mathrm{SAH}$, so we cannot speculate on any other types. In addition, the patient was treated conservatively even with hydrocephalus occurring within a month, if the symptoms were not emergent. Therefore, all cases of hydrocephalus in this study were associated with emergent conditions. The number of cases is still not enough to draw conclusions about the efficacy of ETV on this type of hydrocephalus. However, some characteristics of ETV were revealed.

Seven of the 8 patients could be released from ex- ternal CSF drainage after ETV with stable ventricular size and no further consciousness deterioration, but two patients with IPH did not show improved consciousness. Therefore, ETV performed for hydrocephalus following aneurysmal SAH may achieve control of increased ICP. Physical rehabilitation was initiated within a week after ETV in these 7 patients. Since early rehabilitation is critical for the recovery of elderly patients with consciousness disturbance, ${ }^{11)}$ this is a significant advantage of ETV. Increased ICP due to hydrocephalus following aneurysmal SAH during this period is probably the result of an obstructive mechanism in the ventricular system, and this condition can be resolved with ETV. However, we should note that ICP was monitored in only 3 patients, and whether the reduction of ICP observed after ETV is adequate for full recovery remains unclear.

Hydrocephalus after aneurysmal SAH can be classified into 3 stages, ${ }^{16)}$ with hydrocephalus occurring more than 2 weeks after aneurysmal SAH classified as chronic hydrocephalus. Since external CSF drainage is usually maintained in place for more than a week, acute (0-3 days) and subacute (4-13 days) hydrocephalus cannot be distinguished. We divided the mode of hydrocephalus into "enlarged ventricles" and "drain removal failure," since we speculate that the mode of hydrocephalus may affect the result. "Drain removal failure" can be regarded as hydrocephalus with more acute onset and is more likely to involve the obstructive component, compared with "enlarged ventricles" hydrocephalus, which can be classified as "chronic hydrocephalus."16) However, our findings do not give any insights. More studies are necessary to understand this point.

Four patients showed some cognitive improvement after ETV. This is also an attractive advantage of ETV, but we should be aware that no patient achieved full cognitive recovery after only ETV. Aneurysmal SAH can also cause impaired cognition or other neurological deficits, so whether VP shunt implantation will resolve residual neurological deficits in patients with enlarged ventricles compared to admission is quite difficult to determine. Two patients recovered completely after VP shunt implantation, suggesting only ETV was not sufficient. However, for the two patients who did not improve after VP shunt implantation, ETV seemed to be the essential treatment. Only ETV may alleviate all symptoms in some patients, but they are difficult to identify. How to estimate the effect of VP shunt implantation for ventriculomegaly after aneurysmal SAH has been long discussed, but still no reliable methods have been established. ${ }^{3,10)}$ There- 
fore, we still recommend VP shunting for this type of hydrocephalus when appropriate.

Three of the 8 patients definitely required VP shunt implantation, one for no response to ETV and two for incomplete cognitive impairment, which completely resolved after shunting. The limited number of patients makes common tendencies difficult to determine, but both patients who were treated with endovascular coiling procedures were included in these 3 patients. Hydrocephalus following aneurysmal $\mathrm{SAH}$ is essentially communicating, and washing out SAH during clipping surgery and cisternal drainage placement may reduce the risk of communicating hydrocephalus. Since neither washing out $\mathrm{SAH}$ following craniotomy nor cisternal drainage placement can be performed after the coiling procedure, hydrocephalus in such patients may be difficult to resolve only with ETV.

The timing of VP shunting after aneurysmal SAH has not been established, ${ }^{1)}$ and we are not sure whether one month is adequate for delaying VP shunt implantation. Indeed, delay of VP shunt implantation after aneurysmal SAH is not mandatory, and VP shunting has been performed even within days of aneurysmal SAH. ${ }^{14)}$ An observation period of at least 2 weeks has been recommended, considering the time required to develop fibrosis of the meninges as a consequence of aneurysmal SAH. ${ }^{16)}$ We also believe that a washout period of red blood cells in CSF, which can cause shunt failure, is also desirable.1,2) External drainage placement after aneurysmal surgery is often prolonged in our institute, which may induce bacterial colonization, ${ }^{9)}$ although we did not confirm any cases of meningitis in our study. It is advantageous to wait as long as possible for bacterial elimination before VP shunt implantation. ${ }^{7)}$ Although a VP shunt implanted within one month may have a higher rate of failure, the fenestration made by ETV after aneurysmal SAH also carries a risk of obstruction. ${ }^{13)}$ Therefore, careful observation to detect hydrocephalus recurrence is necessary even after effective ETV, since we found that reclosure of the fenestration can occur within 15 days after ETV.5)

ETV offers an effective option for controlling increased ICP associated with hydrocephalus after aneurysmal SAH, and will also help to improve cognitive function, but this effect is limited. Since whether the maximum resolution is obtained with ETV is hard to confirm, VP shunt implantation should be the main treatment for hydrocephalus with impaired cognitive function. ETV is a temporary intervention in limited conditions, such as during the waiting period for the clearance of aneurysmal SAH.

\section{Acknowledgments}

The authors thank Okayama Neuromedical Research Association at National Hospital Organization Okayama Medical Center for editorial assistance.

\section{References}

1) Baired C, Farner S, Mohr C, Pittman T: The effects of protein, red blood cells and whole blood on PS valve function. Pediatr Neurosurg 37: 186-193, 2002

2) Brydon HL, Bayston R, Hayward R, Harkness W: The effect of protein and blood cells on the flow-pressure characteristics of shunts. Neurosurgery 38: 498-505, 1996

3) Chang CC, Kuwana N, Ito S, Yokoyama T, Kanno H, Yamamoto I: Cerebral haemodynamics in patients with hydrocephalus after subarachnoid haemorrhage due to ruptured aneurysm. Eur J Nucl Med Mol Imaging 30: 123-126, 2003

4) Dorai Z, Hynan LS, Kopitnik TA, Samson D: Factors related to hydrocephalus after aneurysmal subarachnoid hemorrhage. Neurosurgery 52: 763-771, 2003

5) Fukuhara T, Luciano MG, Kowalski RJ: Clinical features of third ventriculostomy failures classified by fenestration patency. Surg Neurol 58: 102-110, 2002

6) Fukuhara T, Vorster SJ, Luciano MG: Risk factors for failure of endoscopic third ventriculostomy for obstructive hydrocephalus. Neurosurgery 46: 11001111,2000

7) Gerner-Smidt P, Stenager E, Kock-Jensen C: Treatment of ventriculostomy-related infections. Acta Neurochir (Wien) 91: 47-49, 1988

8) Komotar RJ, Olivi A, Rigamonti D, Tamargo RJ: Microsurgical fenestration of the lamina terminalis reduced the incidence of shunt-dependent hydrocephalus after aneurysmal subarachnoid hemorrhage. Neurosurgery 51: 1403-1413, 2002

9) Lo CH, Spelman D, Bailey M, Cooper DJ, Rosenfeld JV, Brecknell JE: External ventricular drain infections are independent of drain duration: an argument against elective revision. J Neurosurg 106: 378-383, 2007

10) Ohkuma H, Tanaka M, Kondoh I, Suzuki S: Role of 123I-IMP SPET in the early diagnosis of borderline chronic hydrocephalus after aneurysmal subarachnoid haemorrhage. Eur J Nucl Med 27: 559-565, 2000

11) Saciri BM, Kos N: Aneurysmal subarachnoid haemorrhage: outcomes of early rehabilitation after surgical repair of ruptured intracranial aneurysms. J Neurol Neurosurg Psychiatry 72: 334-337, 2002

12) Sheehan JP, Polin RS, Sheehan JM, Baskaya MK, Kassell NF: Factors associated with hydrocephalus after aneurysmal subarachnoid hemorrhage. Neurosurgery 45: 1120-1128, 1999

13) Siomin V, Cinalli G, Grotenhuis A, Golash A, Oi S, Kothbauer K, Weiner H, Roth J, Beni-Adani L, Pierre- 
Kahn A, Takahashi Y, Mallucci C, Abbott R, Wisoff J, Constantini S: Endoscopic third ventriculostomy in patients with cerebrospinal fluid infection and/or hemorrhage. J Neurosurg 97: 519-524, 2002

14) Steinke D, Weir B, Disney L: Hydrocephalus following aneurysmal subarachnoid haemorrhage. Neurol Res 9: 3-9, 1987

15) Tomasello F, d'Avella D, de Divitiis O: Does lamina terminalis fenestration reduce the incidence of chronic hydrocephalus after subarachnoid hemorrhage? Neurosurgery 45: 827-832, 1999

16) Vale FL, Bradley EL, Fisher WS III: The relationship of subarachnoid hemorrhage and the need for postoperative shunting. J Neurosurg 86: 462-466, 1997

Address reprint requests to: Toru Fukuhara, M.D., Division of Neurosurgery, Neuro-Research Institute for Stroke Care, National Hospital Organization Okayama Medical Center, 1711-1 Tamasu, Okayama 701-1192, Japan.

\section{Commentary}

The authors relate their experience with endoscopic third ventriculostomy following aneurysmal subarachnoid hemorrhage. They indicate that endoscopic third ventriculostomy has limited utility: the majority of patients required subsequent ventricular shunting. Unfortunately, the number of the patients in the present study is small and thus, prognostic factors could not be developed to predict which patients might benefit from this procedure. In pediatric patients, Drake and his associates found that age was strongly correlated with longer term success. ${ }^{1)}$ Personally, I attempt to fenestrate the lamina terminalis during clip ligation of ruptured aneurysms, especially in patients who have intraventricular hemorrhage or a significant amount of blood in their subarachnoid space. In contrast to an endoscopic approach, direct third ventriculostomy appears to lessen the subse- quent need for shunting. ${ }^{2)}$ The present study contributes to our understanding of the limitations of endoscopic third ventriculostomy.

\section{References}

1) Drake JM; Canadian Pediatric Neurosurgery Study Group: Endoscopic third ventriculostomy in pediatric patients: the Canadian experience. Neurosurgery 60: 881-886, 2007

2) Komotar RJ, Olivi A, Rigamonti D, Tamargo RJ: Microsurgical fenestration of the lamina terminalis reduces the incidence of shunt-dependent hydrocephalus after aneurysmal subarachnoid hemorrhage. Neurosurgery 51: 1403-1413, 2002

H. Richard WINN, M.D.

Professor of Neurosurgery and Neuroscience Mount Sinai School of Medicine New York, New York, U.S.A.

Currently, VP shunting is the main method for the treatment of hydrocephalus following aneurysmal subarachnoid hemorrhage (SAH) because malabsorption of cerebrospinal fluid is generally accepted as the cause of this type of hydrocephalus. The authors analyzed the mechanism of hydrocephalus following aneurysmal SAH and found that hydrocephalus occurring within a month may involve an obstructive component. Based on this theory, they suggested that endoscopic third ventriculostomy (ETV) may be used to treat hydrocephalus following aneurysmal SAH, especially hydrocephalus occurring within a month. I think there are two advantages of this suggestion: use of ETV in this period can decrease the risk of infection following external ventricular drainage, and ETV can delay the use of VP shunting to improve the VP shunt patency.

Yazhuo ZHANG, M.D. Deputy Director

Beijing Neurosurgical Institute Beijing, P.R.C. 